\title{
SUBGROUPS OF AMALGAMATED FREE PRODUCTS
}

\author{
BY EDWARD T. ORDMAN ${ }^{1}$
}

Communicated by Michio Suzuki, September 22, 1969

In 1934 Kuroš [5] proved that "a subgroup of a free product of groups is again a free product." Several attempts have been made to extend this to a result about a free product of groups with an amalgamated subgroup, notably [4] and [6]. Theorem 1 here gives to any subgroup of a free product with amalgamated subgroup an induced structure of the same type. We here indicate very briefly the method of proof. Details and related results will appear elsewhere.

Definition 0 . Let $G_{\mu}$ be groups, for $\mu$ in an index set $M$, and let $G$ be a group which is isomorphic to a subgroup of each $G_{\mu}$ under given maps $\delta_{\mu}: G \rightarrow G_{\mu}$. The free product of the groups $G_{\mu}$ with the amalgamated subgroup $G$, denoted $\bar{G}=\left(*_{\mu} G_{\mu}\right)_{G}$, is the factor group of the free product $\left(*_{\mu} G_{\mu}\right)$ with respect to the normal subgroup generated by all elements of the form $\delta_{\mu}(g) \delta_{\nu}(g)^{-1}$, where $g$ runs through $G$ and the pair $(\mu, \nu)$ runs through $M \times M$. That is, $\bar{G}$ is the free product of the $G_{\mu}$ with the subgroups isomorphic to $G$ identified.

Theorem 1. Suppose:

$\bar{G}=\left(*_{\mu} G_{\mu}\right)_{G}$ is a free product of groups with amalgamated subgroup $G, \mu$ in an index set $M$;

$\bar{K}=\left(*_{\mu} K_{\mu}\right)$ is a free product of groups, $\mu \in M$ the same index set; $f: \bar{G} \rightarrow \bar{K}$ is a group homomorphism with $f\left(G_{\mu}\right) \subset K_{\mu}$ for each $\mu$; and $\bar{H}$ is a subgroup of $\bar{G}$ such that $f(\bar{H})=\bar{K}$.

Then:

$\bar{H}$ is expressible as $\left(*_{\mu} H_{\mu}\right)_{H}$ with $f\left(H_{\mu}\right) \subset K_{\mu}$;

$H$ is generated as a subgroup of $G$ by certain subgroups

$$
g_{0 \nu} G_{0 \nu} g_{0 \nu}^{-1}, G_{0 \nu} \subset G_{0}, g_{0 \nu} \in \operatorname{ker} f \subset \bar{G}, \text { for } \nu \text { in an index set } N_{0} ;
$$

Each $H_{\mu}$ is generated as a subgroup of $G$ by certain subgroups

$$
g_{\mu \nu} G_{\mu \nu} g_{\mu \nu}^{-1}, G_{\mu \nu} \subset G_{\mu}, g_{\mu \nu} \in \operatorname{ker} f \subset \bar{G}, \text { for } \nu \text { in an index set } N_{\mu},
$$

AMS Subject Classifications. Primary 2052, 2095; Secondary 2010.

Key Words and Phrases. Free product of groups with amalgamation, amalgamated subgroup, groupoid, covering groupoid.

1 These results are contained in the author's doctoral thesis submitted to Princeton University, written under the direction of Professor J. Stallings at the University of California (Berkeley). This research was supported by a Danforth Graduate Fellowship. 
together with some elements of $G$ of the form $g_{1} g_{2} g_{3}, g_{2} \in G_{\mu}, g_{1}$ and $g_{3} \in$ ker $f$; and finally,

if $G=\{1\}$, then $H=\{1\}$ and each $H_{\mu}$ is the free product of the indicated subgroups together with the free group on the indicated elements: $H_{\mu}=F_{\mu^{*}}\left(*_{\nu} g_{\mu \nu} G_{\mu \nu} g_{\mu \nu}^{-1}\right)$.

Letting $\bar{K}=\{1\}, G=\{1\}$, the Kuroš Subgroup Theorem is an immediate corollary:

Corollary 2. If $\bar{G}=\left(*_{\mu} G_{\mu}\right)$ and $\bar{H} \subset \bar{G}$, then $\bar{H}$ is expressible as $\bar{H}=F *\left(*_{\delta} H_{\delta}\right)$ where $F$ is a free group and each $H_{\delta}$ is conjugate in $\bar{G}$ to a subgroup of some $G_{\mu}$.

If $G=\{1\}, \bar{K} \neq\{1\}$, we have the following theorem of P. J. Higgins [3]:

Corollary 3. Let $\bar{G}=\left(*_{\mu} G_{\mu}\right)$ and $\bar{K}=\left(*_{\mu} K_{\mu}\right)$ be free products of groups. Let $f: \bar{G} \rightarrow \bar{K}$ be a group homomorphism with $f\left(G_{\mu}\right) \subset K_{\mu}$ for each $\mu$. Let $\bar{H}$ be a subgroup of $\bar{G}$ with $f(\bar{H})=\bar{K}$. Then $\bar{H}=\left(*_{\mu} H_{\mu}\right)$ with $f\left(H_{\mu}\right) \subset K_{\mu}$ for each $\mu$.

Higgins gives as a corollary of this the generalization of Gruško's Theorem [2] due to Wagner [8]:

CoRollary 4. Let $g: F \rightarrow\left(*_{\mu} K_{\mu}\right)$ be a map of a free group onto a free product of groups. Then $F$ is itself a free product $F=\left(*_{\mu} F_{\mu}\right)$ with $g\left(F_{\mu}\right) \subset K_{\mu}$.

INDICATION OF PROOF OF ThEOREM 1. The proof makes extensive use of groupoids: we are motivated by the fundamental groupoid of homotopy classes of paths with endpoints fixed in a topological space. In general, a groupoid may be defined as a category in which each map has an inverse. Amalgamated free products of groupoids may be constructed analogously to those of groups. A groupoid has a fundamental (vertex) group and in the case of a free product with a connected amalgamated subgroupoid, the fundamental group of the product is the corresponding product of fundamental groups.

Regard $\bar{G}$ as a groupoid. It has a "covering space," a groupoid $\bar{C}$ whose fundamental group is isomorphic to $\bar{H}[1]$, [3]. The map $\bar{C} \rightarrow \bar{G}$ lets us carry back to $\bar{C}$ a structure as a free product with amalgamated subgroupoid. However, the amalgamated subgroupoid is in general disconnected. We may now use a construction motivated by Stallings' [7] binding tie construction on the induced map $\bar{C} \rightarrow \bar{K}$. That is, we choose certain paths connecting components of the amalgamated subgroupoid of $\bar{C}$ which map to the identity of $\bar{K}$, and 
find an expression of $\bar{C}$ as a free product with an amalgamated subgroupoid that includes these paths. It is these paths that appear as the $g_{0 \nu}, g_{\mu \nu}, g_{1}$, and $g_{3}$ in the statement of the theorem.

One of the lemmas needed in rearranging $\bar{C}$ may be of independent interest as applied to groups.

THEOREM 5. Let $\bar{G}$ be the free product of groups $G_{\mu}$ with amalgamated subgroup $G$, and let $g$ be any element of $\cup G_{\mu} \backslash G$. Denote by $G_{\mu}^{*}$ (respectively $\left.G^{*}\right)$ the subgroup of $\bar{G}$ generated by $G_{\mu}$ (resp. $G$ ) and $\{g\}$. Then $\bar{G}$ is also the free product of the groups $G_{\mu}^{*}$ with amalgamated subgroup $G^{*}$.

Theorem 5 also works for groupoids, and the singleton $\{g\}$ may be replaced by larger sets by induction.

\section{REFERENCES}

1. R. Brown, Elements of modern topology, McGraw-Hill, New York, 1968. MR 37 \#3563.

2. I. Gruško, Über die Basen eines freien Produktes von Gruppen, Mat. Sb. N. S. 8 (50) (1940), 169-182. (Russian) MR 2, 215.

3. P. J. Higgins, Grushko's theorem, J. Algebra 4 (1966), 365-372. MR 34 \#4344.

4. V. Kalašnikov and A. Kuroš, Freie Produkte der Gruppen mit vereinigten Untergruppen der Zentren, Dokl. Akad. Nauk. 6 (1935), 285-286. (Russian) Zentralblatt 11, 151.

5. A. Kuroš, Die Untergruppen der freien Produkte von beliebigen Gruppen, Math. Ann. 109 (1934), 647-660.

6. Hanna Neumann, Generalized free products with amalgamated subgroups. I, Amer. J. Math. 70 (1948), 590-625; II, Amer. J. Math. 71 (1949), 491-540. MR 10, 233; MR 11, 8.

7. J. R. Stallings, A topological proof of Grushko's theorem on free products, Math. Z. 90 (1965), 1-8. MR 32 \#5723.

8. D. H. Wagner, On free products of groups, Trans. Amer. Math. Soc. 84 (1957), 352-378. MR 18, 790.

University of KentuCKy, Lexington, Kentucky 40506 